\title{
Paired-Pulse Depression at Photoreceptor Synapses
}

\author{
Katalin Rabl, ${ }^{1}$ Lucia Cadetti, ${ }^{1}$ and Wallace B. Thoreson ${ }^{1,2}$ \\ Departments of ${ }^{1}$ Ophthalmology and Visual Sciences and ${ }^{2}$ Pharmacology and Experimental Neuroscience, University of Nebraska Medical Center, Omaha, \\ Nebraska 68198-5840
}

Synaptic depression produced by repetitive stimulation is likely to be particularly important in shaping responses of second-order retinal neurons at the tonically active photoreceptor synapse. We analyzed the time course and mechanisms of synaptic depression at rod and cone synapses using paired-pulse protocols involving two complementary measurements of exocytosis: (1) paired whole-cell recordings of the postsynaptic current (PSC) in second-order retinal neurons and (2) capacitance measurements of vesicular membrane fusion in rods and cones. PSCs in ON bipolar, OFF bipolar, and horizontal cells evoked by stimulation of either rods or cones recovered from paired-pulse depression (PPD) at rates similar to the recovery of exocytotic capacitance changes in rods and cones. Correlation between presynaptic and postsynaptic measures of recovery from PPD suggests that $80-90 \%$ of the depression at these synapses is presynaptic in origin. Consistent with a predominantly presynaptic mechanism, inhibiting desensitization of postsynaptic glutamate receptors had little effect on PPD. The depression of exocytotic capacitance changes exceeded depression of the presynaptic calcium current, suggesting that it is primarily caused by a depletion of synaptic vesicles. In support of this idea, limiting $\mathrm{Ca}^{2+}$ influx by using weaker depolarizing stimuli promoted faster recovery from PPD. Although cones exhibit much faster exocytotic kinetics than rods, exocytotic capacitance changes recovered from PPD at similar rates in both cell types. Thus, depression of release is not likely to contribute to differences in the kinetics of transmission from rods and cones.

Key words: ribbon synapse; capacitance measurement; retina; tiger salamander; photoreceptor; postsynaptic current

\section{Introduction}

Rod and cone photoreceptors maintain relatively depolarized resting potentials ( -35 to $-45 \mathrm{mV}$ in darkness) allowing the continuous release and recycling of glutamate-laden vesicles. Repetitive presynaptic stimulation produces a depression of postsynaptic responses at many ribbon and conventional synapses (Kusano and Landau, 1975; von Gersdorff and Matthews, 1997; Wu and Borst, 1999) (for review, see Zucker and Regehr, 2002). Similarly, pairs of depolarizing pulses depress postsynaptic responses at the synapses from cone photoreceptors onto OFF bipolar cells (DeVries, 2000). The extent and kinetics of postsynaptic depression produced by the continuous release of glutamate from photoreceptors are likely to shape the responses of secondorder retinal neurons. We analyzed the time course and mechanisms of synaptic depression at both rod and cone synapses using paired-pulse protocols involving two complementary measurements of exocytosis: (1) paired whole-cell recordings of the postsynaptic current (PSC) in second-order retinal neurons and (2) capacitance measurements of vesicular membrane fusion in rods and cones.

Both presynaptic and postsynaptic factors can contribute to postsynaptic depression. Presynaptic factors include regulation

\footnotetext{
Received Aug. 30, 2005; revised Jan. 12, 2006; accepted Jan. 13, 2006.

This work was supported by the National Eye Institute (EY-10542), Fight for Sight, and Research to Prevent Blindness. We thank Eric Bryson for his technical assistance.

Correspondence should be addressed to Wallace B. Thoreson, Ophthalmology Department, University of Nebraska Medical Center, Durham Research Center, Room 4026, Omaha, NE 68198-5840. E-mail: wbthores@unmc.edu.

D0I:10.1523/JNEUROSCI.3667-05.2006

Copyright $\odot 2006$ Society for Neuroscience $\quad$ 0270-6474/06/262555-09\$15.00/0
}

of calcium currents $\left(I_{\mathrm{Ca}}\right)$ (DeVries, 2001; Rabl and Thoreson, 2002; Hosoi et al., 2005) and depletion of the pool of readily releasable vesicles from the presynaptic terminal (Charlton et al., 1982; von Gersdorff and Matthews, 1997; Schneggenburger et al., 2002). Postsynaptically, paired-pulse depression (PPD) can be produced by slow recovery of glutamate receptors from desensitization (Otis et al., 1996b; DeVries, 2000; Rozov et al., 2001). Our results suggest that receptor desensitization plays a minor role in PPD at most rod and cone synapses and that a substantial fraction of the PPD in synaptic transmission from photoreceptors is caused by a presynaptic depression of release. The depression of presynaptic release greatly exceeded depression of the presynaptic $I_{\mathrm{Ca}}$, suggesting that it is primarily caused by a depletion of synaptic vesicles. Although depolarization evokes a faster and more transient release of vesicles from cones than rods (Rabl et al., 2005), we found no difference in the rates of vesicle replenishment by rods and cones. This suggests that depression of release is not likely to contribute to differences in the impulse response characteristics of synaptic transmission from rods and cones (Schnapf and Copenhagen, 1982).

\section{Materials and Methods}

Experiments were performed using aquatic tiger salamanders (Ambystoma tigrinum), $18-25 \mathrm{~cm}$ in length. Animals were handled according to protocols approved by the University of Nebraska Medical Center Institutional Animal Care and Use Committee. The salamander was decapitated, and the brain and spinal cord were rapidly pithed. After enucleation, the anterior segment of the eye, including the lens, was removed. The eyecup was cut into quarters, and a section of eyecup was placed vitreal side down on a piece of filter paper $(2 \times 5 \mathrm{~mm}$; type AAWP; 0.8 $\mu \mathrm{m}$ pores; Millipore, Bedford, MA). The isolated retina was cut into 
slices $(125 \mu \mathrm{m}$ ) with a razor blade tissue chopper (Stoelting, Wood Dale, IL). Slices were positioned in the recording chamber to allow viewing of the retinal layers when viewed with a water immersion objective [ $40 \times$, 0.7 numerical aperture (NA) or $60 \times, 1.0 \mathrm{NA}$ ] on an upright fixed-stage microscope (BHWI, Olympus, Tokyo, Japan; or E600 FN, Nikon, Tokyo, Japan). Slices were superfused at $\sim 1 \mathrm{ml} / \mathrm{min}$.

For recordings of capacitance changes, the superfusate contained the following (in mM): $95 \mathrm{NaCl}, 2.5 \mathrm{KCl}, 1.8 \mathrm{CaCl}_{2}, 0.5 \mathrm{MgCl}_{2}, 5 \mathrm{CsCl}, 10$ tetraethylammonium chloride (TEACl), 10 HEPES, 5 glucose, and 0.2 niflumic acid, pH 7.8. Niflumic acid, TEACl, and $\mathrm{CsCl}$ were included to minimize voltage and $\mathrm{Ca}^{2+}$-activated conductances and their possible intrusion into $C_{\mathrm{m}}$ measurements. For recordings of postsynaptic currents, slices were superfused with a solution containing the following (in mm): $111 \mathrm{NaCl}, 2.5 \mathrm{KCl}, 1.8 \mathrm{CaCl}_{2}, 0.5 \mathrm{MgCl}_{2}, 10$ HEPES, 5 glucose, 0.001 strychnine, and 0.1 picrotoxin, $\mathrm{pH}$ 7.8. Solution osmolarity was tested with a vapor pressure osmometer (Wescor, Logan, UT) and adjusted, if necessary, to $\sim 242 \mathrm{mOsm}$. The presence of HEPES minimized effects of vesicular protons in the synaptic cleft (DeVries, 2001; Hosoi et al., 2005). Unless otherwise specified, chemicals were obtained from Sigma (St. Louis, MO).

Details of capacitance measurements, paired whole-cell recordings, and passive cell membrane properties are described in detailed by Rabl et al. (2005) and summarized briefly below. Whole-cell recordings were obtained using $8-15 \mathrm{M} \Omega$ patch electrodes pulled from borosilicate glass (outer diameter, $1.2 \mathrm{~mm}$; inner diameter, $0.95 \mathrm{~mm}$; with internal filament; World Precision Instruments, Sarasota, FL) on a PP-830 micropipette puller (Narishige, East Meadow, NY). The pipette solution contained the following (in $\mathrm{mM}$ ): 94 Cs-gluconate, $9.4 \mathrm{TEACl}, 1.9 \mathrm{MgCl}_{2}, 9.4$ MgATP, 0.5 GTP, 5 EGTA, and 32.9 HEPES, pH 7.2.

For capacitance recording, pipettes were coated with Sylgard (Dow Corning, Midland, MI) to reduce stray capacitance. Residual pipette capacitance was compensated electronically. Capacitance measurements were made using the "track-in" mode of the Optopatch patch-clamp amplifier (Johnson et al., 2002). The holding potential was varied sinusoidally ( $600 \mathrm{~Hz} ; 30 \mathrm{mV}$ peak to peak) about a holding potential of -70 $\mathrm{mV}$. Data were acquired and analyzed with a Digidata 1200 interface and pClamp 8.1 software (Molecular Devices, Union City, CA). Lock-in signals were gated out during the test step and for $3 \mathrm{~ms}$ afterward.

For paired recordings, rods or cones were voltage clamped simultaneously with adjacent postsynaptic neurons using a Multiclamp patchclamp amplifier (Molecular Devices) or an Optopatch amplifier (Cairn Instruments, Faversham, UK) paired with an Axopatch 200B (Molecular Devices). Both recording pipettes were positioned with Huxley-Wall micromanipulators (Sutter Instruments, Novato, CA). Currents were acquired using Digidata 1322 or 1200B interfaces and pClamp 8.1 software (Molecular Devices). Rods and cones were clamped at $-70 \mathrm{mV}$, bipolar cells at $-50 \mathrm{mV}$, and horizontal cells at $-40 \mathrm{mV}$. PSC amplitude was measured relative to the baseline current level before the first test pulse. Horizontal, ON bipolar, and OFF bipolar cells were distinguished by their response characteristics (Thoreson et al., 1997) and appearance after staining with Lucifer yellow (2 mg/ml) or AlexaFluor 568 (0.2 mg/ $\mathrm{ml}$; Invitrogen, San Diego, CA). Charging curves of rods, cones, bipolar, and horizontal cells in the retinal slice preparation can typically be fit by single exponential functions (Cadetti et al., 2005; Rabl et al., 2005). Single exponential fits to horizontal cell charging curves suggest that the cells were mostly uncoupled from their neighbors in retinal slices. The finding that horizontal cell EPSCs reverse at $\sim 0 \mathrm{mV}$ also suggests that adequate space clamp can be maintained (Cadetti and Thoreson, 2006).

To evoke PPD, we applied two identical test pulses using interstimulus intervals ranging from $100 \mathrm{~ms}$ to $30 \mathrm{~s}$. In most experiments, strong depolarizing test steps from -70 to $-10 \mathrm{mV}(100 \mathrm{~ms})$ were used to elicit maximal responses. Some experiments were also done with steps to -30 $\mathrm{mV}$. The $100 \mathrm{~ms}$ duration of the test step empties the readily releasable pool of vesicles in rods and cones (Thoreson et al., 2004; Rabl et al., 2005). We waited at least $30 \mathrm{~s}$ between trials. The presentation sequence was varied between cells to avoid possible order effects. For trials with caged glutamate, 4-methoxy-7-nitroindolinyl-caged L-glutamate (MNI-glutamate; Tocris Bioscience, Ellisville, $\mathrm{MO}$ ) was bath applied and photolyzed by $1 \mathrm{~ms}$ flashes of UV light derived from a xenon arc flash lamp (Rapp

\section{A. Rod - ON bipolar}
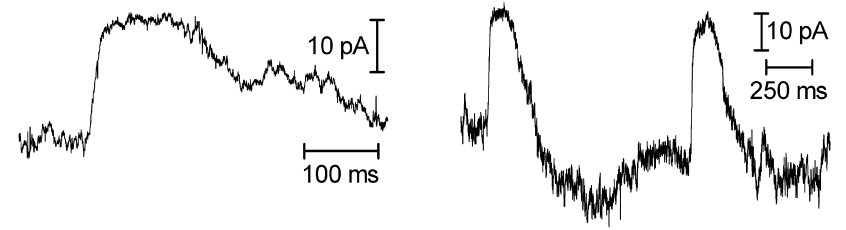

\section{B. Rod - OFF bipolar}
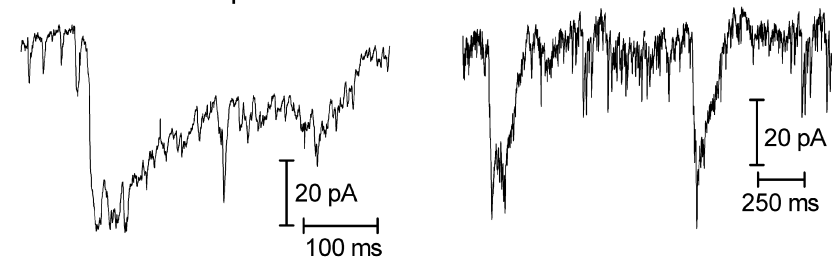

\section{Rod - horizontal cell}
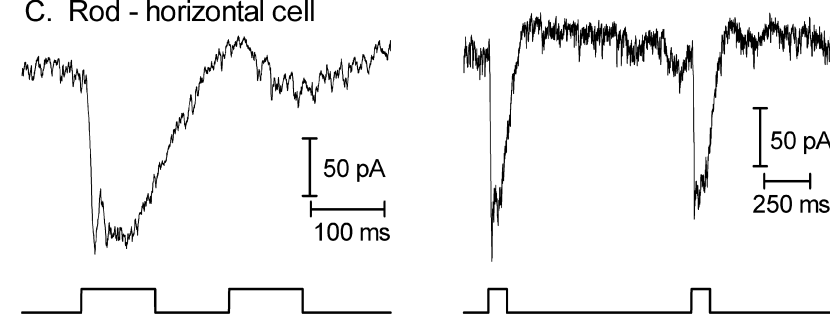

Figure 1. Repetitive stimuli depressed PSCs evoked by presynaptic stimulation of rods in all three types of second-order retinal neurons. PSCs were evoked by depolarizing steps ( -70 to $-10 \mathrm{mV}, 100 \mathrm{~ms}$ ) applied to rods while simultaneously recording from ON bipolar $(\boldsymbol{A})$, OFF bipolar $(\boldsymbol{B})$, and horizontal $(\boldsymbol{C})$ cells. An interpulse interval of $100 \mathrm{~ms}$ (left) substantially depressed subsequent PSCS, whereas much less depression was evident with an interpulse interval of $1 \mathrm{~s}$ (right).

Optoelectronic, Hamburg, Germany) coupled through the epifluorescence port of the microscope (E600FN; Nikon). Flash intensity was chosen to evoke a near-maximal amplitude response.

The criterion for statistical significance was chosen to be $p<0.05$ and evaluated with $F$ tests or Student's $t$ tests using Prism 4.0 (GraphPad Software, San Diego, CA). Variability is reported as \pm SEM.

\section{Results}

Paired-pulse depression at the photoreceptor synapse was produced by applying a pair of identical depolarizing pulses $(-70$ to $-10 \mathrm{mV}, 100 \mathrm{~ms}$ ) to a photoreceptor while simultaneously recording the PSCs in bipolar or horizontal cells. Test pulses evoked outward PSCs in ON bipolar cells as a result of the closure of cation channels accompanying activation of the metabotropic glutamate receptor mGluR6 (Nawy and Jahr, 1990) and inward PSCs in OFF bipolar and horizontal cells as a result of the activation of non-NMDA ionotropic glutamate receptors (Yang et al., 1998; Maple et al., 1999; Cadetti et al., 2005) (Fig. 1). Stimulating rod photoreceptors with an interval of $100 \mathrm{~ms}$ between test pulses produced a marked depression of PSCs in all three types of second-order retinal neurons: ON bipolar, OFF bipolar, or horizontal cells (Fig. 1). Longer interpulse intervals produced less PPD and almost complete recovery was observed when the interpulse interval was lengthened to $1 \mathrm{~s}$ (Fig. 1).

Stimulating cones with pairs of depolarizing steps also produced a depression of PSCs in bipolar and horizontal cells (Fig. 2). Because of their ability to release glutamate more rapidly than rods (Rabl et al., 2005; Cadetti et al., 2005), EPSCs in cone-driven OFF bipolar and horizontal cells are faster and more transient than those in rod-driven neurons. However, similar to roddriven synapses, evoking PSCs by stimulating cones with an in- 


\section{A. Cone - ON bipolar}
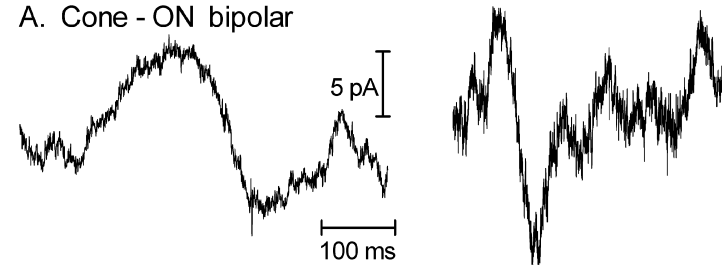

B. Cone - OFF bipolar
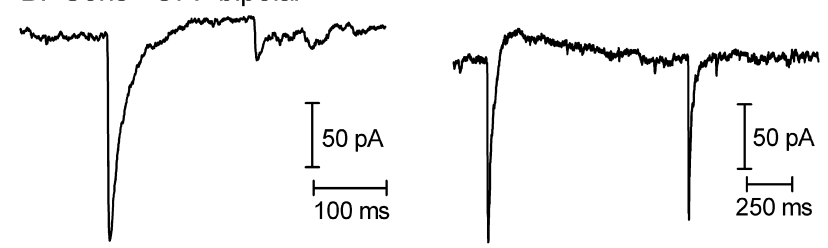

C. Cone - horizontal cell
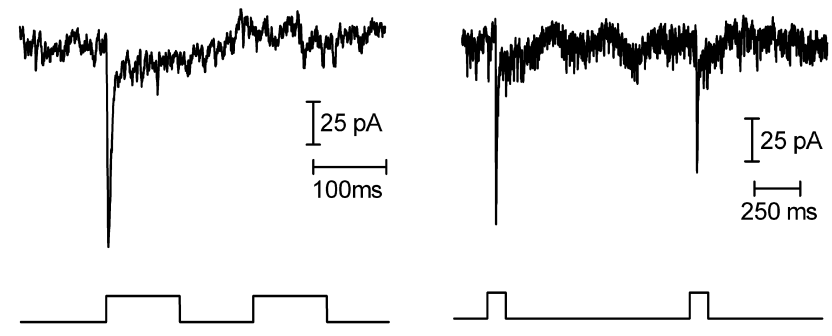

Figure 2. Repetitive stimuli depressed PSCs evoked by presynaptic stimulation of cones in all three types of second-order retinal neurons. PSCs were evoked by depolarizing steps ( -70 to $-10 \mathrm{mV}, 100 \mathrm{~ms}$ ) applied to cones while simultaneously recording from $0 \mathrm{~N}$ bipolar $(\boldsymbol{A})$, OFF bipolar (B), and horizontal $(\boldsymbol{C})$ cells. An interpulse interval of $100 \mathrm{~ms}$ (left) substantially depressed subsequent PSCs, whereas less depression was evident with an interpulse interval of $1 \mathrm{~s}$ (right).
A. Rod-driven cells

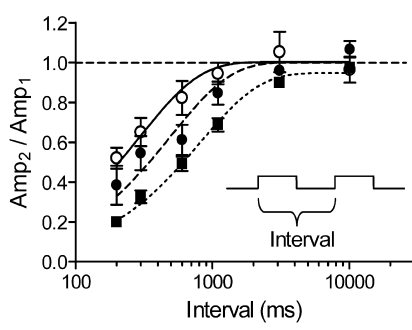

B. Cone-driven cells

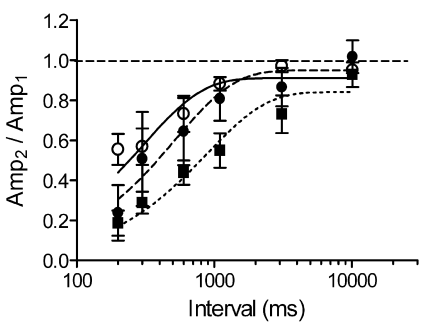

Figure 3. Time course of PSC recovery from PPD. The amplitude of the PSC evoked by the second pulse $\left(\mathrm{Amp}_{2}\right)$ was divided by the amplitude of the first PSC $\left(\mathrm{Amp}_{1}\right)$ and plotted against the time from the beginning of the first pulse to the beginning of the second pulse (inset). Recovery from PPD was fit with single exponential functions. $\boldsymbol{A}$, Rod/ON bipolar PSCs (open circles) recovered more quickly ( $\tau=301 \mathrm{~ms}$ ) than rod/OFF bipolar PSCs (filled circles; $\tau=501$ $\mathrm{ms}$ ), which in turn recovered more rapidly than horizontal cell PSCs (filled squares; $\tau=813 \mathrm{~ms}$ ). $\boldsymbol{B}$, Cone/ON bipolar PSCs (open circles; $\tau=304 \mathrm{~ms}$ ) recovered more quickly than cone/0FF bipolar PSCs (filled circles; $\tau=517 \mathrm{~ms}$ ), which in turn recovered more rapidly than horizontal cell PSCs (filled squares; $\tau=884 \mathrm{~ms}$ ). Note that PSCs in the same postsynaptic cell type recovered at similar rates whether driven presynaptically by rods $(\boldsymbol{A})$ or cones $(\boldsymbol{B})$. Rod/ON bipolar, $n=5-7$ data points at every time interval; rod/OFF bipolar, $n=6$; rod/horizontal cell, $n=$ 31-34; cone/0N bipolar, $n=4-6$; cone/0FF bipolar, $n=3$; cone/horizontal cell, $n=7-10$. Error bars represent SEM.

terstimulus interval of $100 \mathrm{~ms}$ produced strong depression of the second response in all three cell types, and PSCs showed substantial recovery with an interpulse interval of $1 \mathrm{~s}$.

The rates of recovery of PSCs among different cell types stimulated by rods or cones are compared in Figure 3. The amplitude of the second PSC (measured from the baseline current level) was divided by the amplitude of the first PSC and plotted against the interval from the beginning of the first pulse to the beginning of the second pulse (i.e., the interval between measurements) (Fig. $3 A$, inset). To quantify the rate of recovery, data were fit with single exponential functions. The best fit function did not return to baseline in cone-driven cells and rod-driven horizontal cells, suggesting there may be an additional slower component that accounts for up to $15 \%$ of the recovery. ON bipolar cell PSCs evoked by rods $(\tau=301 \mathrm{~ms})$ or cones $(\tau=304 \mathrm{~ms})$ recovered more rapidly than OFF bipolar cell PSCs (rod-driven, $\tau=501$ ms; cone-driven, $\tau=517 \mathrm{~ms}$ ), which in turn recovered more rapidly than horizontal cell PSCs (rod-driven, $\tau=813 \mathrm{~ms}$; conedriven, $\tau=884 \mathrm{~ms}$ ). Differences in the time constants of recovery between rod-driven horizontal, ON bipolar, and OFF bipolar cells were significant ( $F$ test, $p<0.0001)$ as were differences between cone-driven cells ( $p=0.0022)$. However, differences in the time constants of recovery among different cell types were not significant when recovery was calculated as a fraction of the depressed current rather than a fraction of the total current (roddriven cells, $p=0.12$; cone-driven cells, $p=0.35$; data not shown), suggesting that these differences are at least partly because of differences in the amount of PPD. The time constants for recovery of PSCs in any one postsynaptic cell type did not differ significantly whether stimulated by rods or cones (rod/horizontal cell vs cone/horizontal cell, $p=0.17$; rod/ON bipolar cell vs cone/ON bipolar cell, $p=0.26$; rod/OFF bipolar cell vs cone/OFF bipolar cell, $p=0.93$ ). These data are consistent with results presented later showing similar rates of recovery from PPD for exocytotic capacitance changes at rod and cone synapses. Although rod- and cone-driven synapses exhibited generally similar rates of recovery, results from one experiment showed an exception to this rule. In this experiment, we recorded from a rod-horizontal cell pair and then switched the presynaptic recording pipette to record from a cone while maintaining the horizontal cell recording. EPSCs in this horizontal cell recovered from PPD evoked by stimulation of the rod much more rapidly than from PPD evoked by stimulation of the cone indicating differences at individual rod and cone synapses contacting this cell.

Differences in the amount of PPD among different postsynaptic cell types are consistent with possible postsynaptic contributions to PPD at rod and cone synapses. An important postsynaptic contributor to PPD at a number of synapses is the desensitization of postsynaptic glutamate receptors (DeVries, 2000; Rozov et al., 2001). Glutamate receptors in OFF bipolar and horizontal cells of the salamander retina are mostly of the AMPA subtype (Yang et al., 1998; Maple et al., 1999; Cadetti et al., 2005). To examine the contribution of desensitization to PPD at photoreceptor synapses, we focused on rod/horizontal cell pairs because it is easier to obtain long stable recordings from rods and because horizontal cells showed the greatest amount of PPD. To test whether desensitization persists long enough to contribute to PPD with interstimulus intervals used in our study, we applied two sequential pulses of glutamate by flash photolysis of a caged glutamate compound, MNI-glutamate ( $1 \mathrm{~mm}$ ), added to the bathing medium. As illustrated in Figure $4 A$, photolysis of caged glutamate with a $1 \mathrm{~ms}$ UV flash produced large transient inward currents in horizontal cells as a result of the abrupt elevation of glutamate levels. A second pulse of glutamate produced by a flash applied $500 \mathrm{~ms}$ later evoked a slightly smaller current averaging $87 \pm 2 \%(n=5)$ of the first. Although the exact time course of glutamate removal from the cleft is not known, this result suggests that as much as 13 of the $51 \%$ depression of rod-driven 

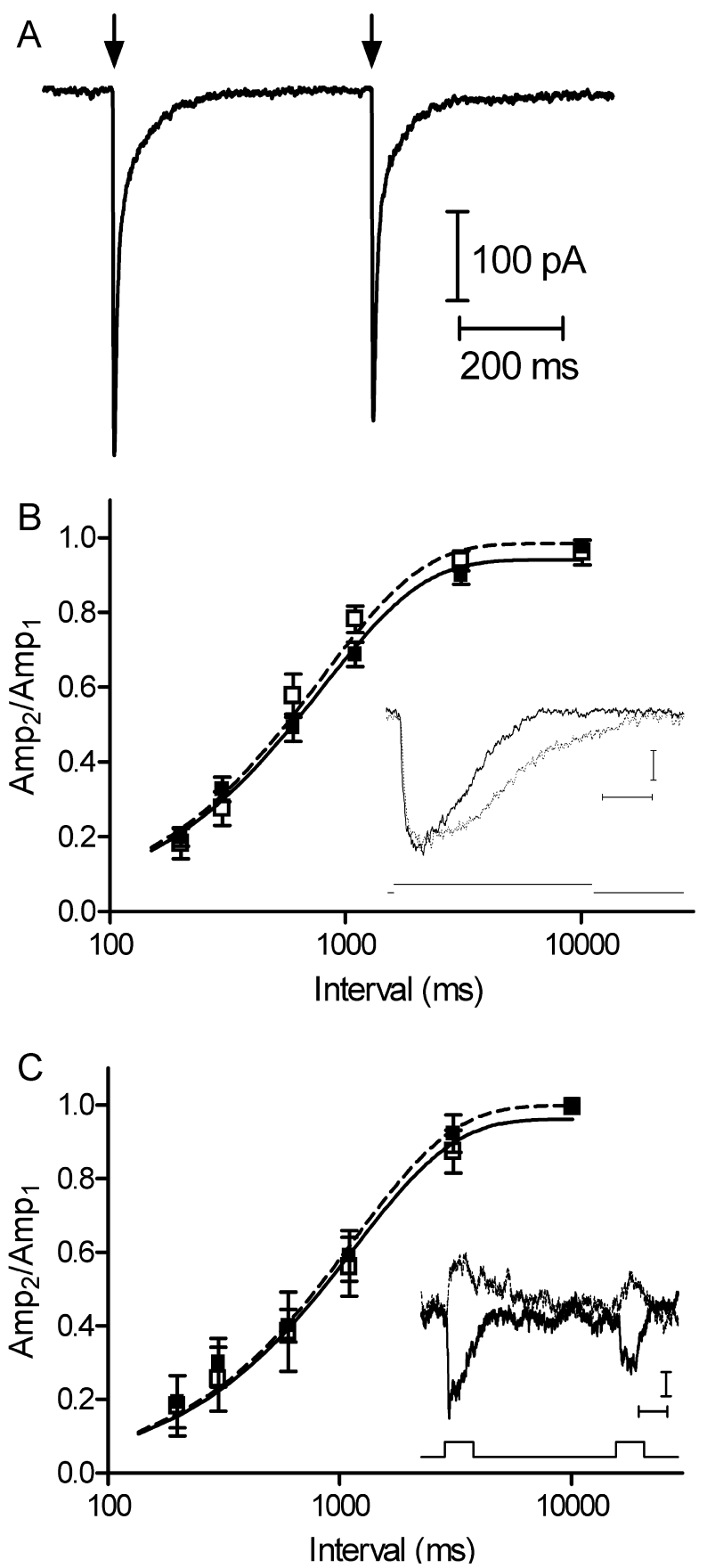

Figure 4. Glutamate receptor desensitization contributes minimally to PPD. A, Inward current evoked in a horizontal cell by flash photolysis of MNI-glutamate $(1 \mathrm{mM})$ showed very little desensitization after an interval of $500 \mathrm{~ms}$. The arrows indicate timing of the flashes. $\boldsymbol{B}$, In a separate experiment, blocking AMPA receptor desensitization with cyclothiazide $(0.1 \mathrm{~mm} ; n=$ 11-14 at each data point) produced little or no change in the recovery from PPD in horizontal cells stimulated by depolarizing steps (to $-10 \mathrm{mV}$ ) applied to presynaptic rods. Control measurements, filled squares, solid line, $\tau=818 \mathrm{~ms}$; cyclothiazide, open squares, dashed line, $\tau=$ $731 \mathrm{~ms}$ (Ftest comparison of $\tau$ values in (TZ and control, $p=0.336$ ). The inset shows an overlay of EPSCs evoked in a horizontal cell by a depolarizing step ( -70 to $-10 \mathrm{mV}, 200 \mathrm{~ms}$ ) applied before (thick trace) and after addition of cyclothiazide (thin dotted trace). Calibration: $50 \mathrm{~ms}, 25$ pA. C, In a third experiment, reducing AMPA receptor desensitization by holding horizontal cells at $+60 \mathrm{mV}$ (open squares; dashed line; $\tau=1210 \mathrm{~ms}$ ) did not alter recovery from PPD produced when the same cells were held at $-60 \mathrm{mV}$ (filled squares; solid line; $\tau=1094 \mathrm{~ms} ; F$ test comparison of $\tau$ values, $p=0.207$ ). PPD was evoked by pairs of depolarizing steps ( -70 to $-10 \mathrm{mV}$ ) applied to simultaneously recorded presynaptic rods $(n=6)$. The inset shows EPSCS evoked by pairs of stimuli ( -70 to $-10 \mathrm{mV}, 100 \mathrm{~ms}$ ) separated by $500 \mathrm{~ms}$ interval with the horizontal cell held at $-60 \mathrm{mV}$ (thick trace) and $+60 \mathrm{mV}$ (thin dotted trace). Calibration: 25 $\mathrm{ms}, 25 \mathrm{pA}$. Error bars represent SEM. horizontal cell responses observed with $500 \mathrm{~ms}$ interstimulus intervals may be because of glutamate receptor desensitization.

Consistent with the presence of AMPA receptors in horizontal cells (Yang et al., 1998; Cadetti et al., 2005), selectively blocking AMPA receptor desensitization with cyclothiazide $(0.1 \mathrm{~mm})$ (Partin et al., 1993) broadened rod-driven EPSCs in horizontal cells (Fig. $4 B$, inset). In this example, cyclothiazide did not increase the amplitude of the EPSC, although it did so in many other cases (Cadetti et al., 2005). However, cyclothiazide did not significantly accelerate recovery from PPD among rod-driven horizontal cells (Fig. 4B). (An example of PPD recorded in the presence of cyclothiazide is also shown in Fig. 6.) With interstimulus intervals of 0.5 and $1 \mathrm{~s}$, cyclothiazide reduced PPD by $<10 \%$. No differences in PPD were evident with shorter intervals, although it is possible that small changes may have been obscured by the broad EPSC waveform produced by cyclothiazide.

In addition to blocking glutamate receptor desensitization, cyclothiazide has been reported to have presynaptic effects on release in CNS neurons (Diamond and Jahr, 1995; Bellingham and Walmsley, 1999). Glutamate receptor desensitization can also be reduced by voltage clamping the postsynaptic cell at a depolarized membrane potential (Raman and Trussell, 1995; Otis et al., 1996a). Consistent with a membrane potential above 0 $\mathrm{mV}$ and reduced desensitization, holding a horizontal cell at +60 $\mathrm{mV}$ produced an outward EPSC with a broader waveform than that evoked when the cell was voltage clamped at $-60 \mathrm{mV}$ (Fig. $4 C$, inset). In agreement with conclusions from cyclothiazide experiments that desensitization contributes very little to PPD in horizontal cells, PPD recovered at similar rates whether horizontal cells were held at -60 or $+60 \mathrm{mV}$ (Fig. 4C).

A major cause of PPD at many synapses is presynaptic depression of release (Zucker and Regehr, 2002). If multiple vesicles are released at the synapse and release is depressed during the second response, then glutamate levels attained in the synaptic cleft during the second EPSC should be lower than those attained during the first EPSC (Wadiche and Jahr, 2001). To examine glutamate levels during the EPSC, we used a low-affinity, competitive glutamate receptor antagonist, kynurenic acid. In response to a transient elevation of glutamate, kynurenic acid molecules will be displaced by higher-affinity glutamate molecules. The efficacy of kynurenic acid in blocking an EPSC is therefore determined by the kinetics and concentration of glutamate in the cleft (Clements, 1996). Assuming similar kinetics, a low concentration of glutamate will be blocked more strongly by kynurenic acid than a high concentration. Kynurenic acid $(0.5 \mathrm{~mm})$ reduced the amplitude of the initial EPSC in rod-driven horizontal cells to $41 \pm 3 \%$ of control (Fig. $5 \mathrm{~A}$, inset) and significantly increased PPD when compared with control trials conducted in the same cell pairs (Fig. 5A). The more efficacious block of the second EPSC by kynurenic acid is consistent with a lower concentration of glutamate being attained in the synaptic cleft (Hashimoto and Kano, 1998; Wadiche and Jahr, 2001; Shen et al., 2002), but these results can also be interpreted as a relief of postsynaptic receptor saturation during the first EPSC (Foster et al., 2002; Harrison and Jahr, 2003). These two possible interpretations are considered in the Discussion in greater detail.

Presynaptic depression can be the result of vesicle depletion (Zucker and Regehr, 2002). If PPD at the photoreceptor synapse involves the depletion of synaptic vesicles, then postsynaptic cells should exhibit a more rapid recovery from PPD when fewer vesicles are released. Applying depolarizing steps to $-40 \mathrm{mV}$ in rods, the amplitude of EPSCs evoked in postsynaptic horizontal cells 

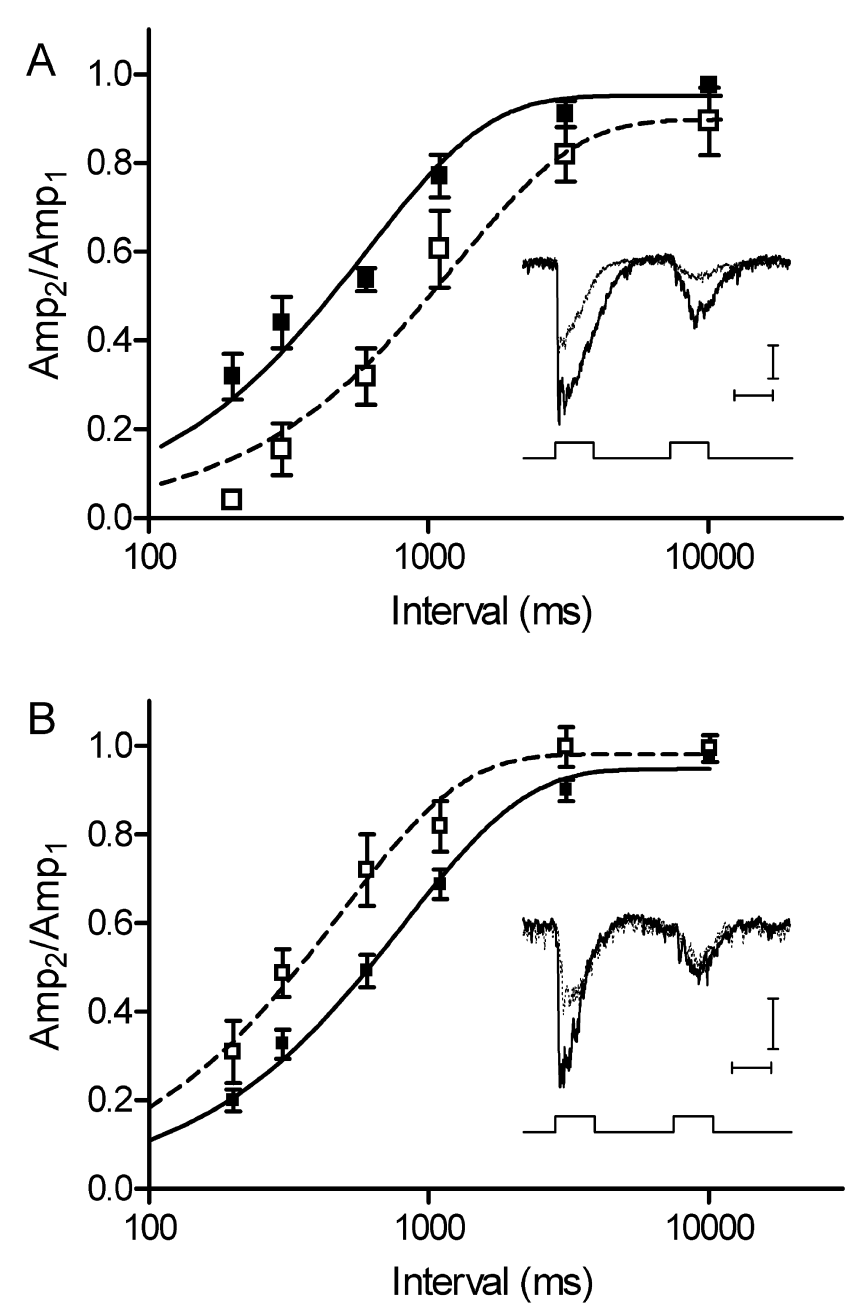

Figure 5. PPD involves presynaptic depression of release. $A$, The low-affinity glutamate receptor antagonist kynurenic acid $(0.5 \mathrm{~mm})$ slowed recovery from PPD of EPSCS recorded from horizontal cells and stimulated by depolarizing steps ( -70 to $-10 \mathrm{mV}, 100 \mathrm{~ms}$ ) applied to presynaptic rods. Kynurenic acid, open squares, dashed line, $\tau=1240 \mathrm{~ms}$; control measurements in the same cell pairs, filled squares, solid line, $\tau=602 \mathrm{~ms}$ (Ftest comparison of $\tau$ values in kynurenic acid and control, $p=0.0002$ ). The inset shows horizontal cell EPS(s in control conditions (large thick trace) and after application of kynurenic acid (smaller thin dotted trace). Calibration: $50 \mathrm{pA}, 50 \mathrm{~ms}$. B, When fewer vesicles were depleted from rod terminals by using mild depolarizing steps to $-40 \mathrm{mV}$ to evoke PPD in horizontal cells ( $n=9$; open squares; dashed line; $\tau=483 \mathrm{~ms}$ ), recovery was faster than when PPD was produced using steps to $-10 \mathrm{mV}$ (filled squares; solid line; $\tau=818 \mathrm{~ms} ;$ F test comparison of $\tau$ values, $p=0.0002$ ). The inset illustrates pairs of horizontal cell EPSCs evoked by depolarizing steps applied to a rod separated by $200 \mathrm{~ms}$ interval. The larger thick trace shows EPSCs evoked by steps to $-10 \mathrm{mV}$, and the smaller thin dotted trace shows EPSCs evoked by steps to $-40 \mathrm{mV}$. Calibration: $50 \mathrm{pA}$, $50 \mathrm{~ms}$. Error bars represent SEM. Amp ${ }_{1}$, Amplitude of the first response; Amp ${ }_{2}$, amplitude of the second response.

averaged $53 \pm 3 \%(n=9)$ of that evoked by steps to $-10 \mathrm{mV}$ in the same cell pairs, indicating that fewer vesicles were released by the weaker depolarizing stimulus (Fig. 5B, inset). Consistent with a depletion model, recovery from PPD was significantly faster when rods were stimulated by steps to $-40 \mathrm{mV}(n=9)$ than when evoked by steps to $-10 \mathrm{mV}$ (Fig. $5 B$ ).

Another possible mechanism for presynaptic depression involves the activation of presynaptic mGluRs. Type III mGluRs have been shown to reduce output from rods by mechanisms that do not involve inhibition of the presynaptic $I_{\mathrm{Ca}}$ (Hirasawa et al., 2002). We compared PPD at the synapse from rods to horizontal cells in the presence or absence of the type III mGluR agonist
L-2-amino-4-phosphonobutyric acid (L-AP-4; $50 \mu \mathrm{M}$ ) and found that L-AP-4 did not significantly alter PPD $(n=3$; data not shown). L-AP-4 can also inhibit $I_{\mathrm{Ca}}$ in cones; the possible contribution of changes in $I_{\mathrm{Ca}}$ to PPD is addressed later in this paper.

Evidence from experiments described above suggested that PPD at the photoreceptor synapse is mostly caused by presynaptic depression of release. We directly examined presynaptic depression of release by measuring membrane capacitance increases accompanying exocytotic fusion of presynaptic vesicles. To avoid artifacts associated with large conductance changes, capacitance measurements were suspended during the step and resumed 3 ms after the step. As detailed by Rabl et al. (2005), tests with a model cell circuit and experiments showing that depolarization-evoked capacitance changes were not correlated with changes in membrane current or access resistance indicate that measured capacitance increases reflected true exocytotic increases in membrane capacitance. Figure 6 shows an experiment in which we directly compared PPD presynaptically and postsynaptically by recording the presynaptic capacitance changes in a rod while simultaneously recording EPSCs in a horizontal cell. Recordings were made in the presence of cyclothiazide $(0.1 \mathrm{~mm})$. Both the depolarization-evoked capacitance increase (Fig. 6A) and EPSC (Fig. $6 B$ ) were markedly depressed when test pulses ( $100 \mathrm{~ms},-70$ to $-10 \mathrm{mV}$ ) were separated by $100 \mathrm{~ms}$. After increasing the interpulse interval to $1 \mathrm{~s}$, both the presynaptic exocytotic response and PSC showed nearly complete recovery. The parallel between recovery of the presynaptic capacitance response and the PSC suggests that much of the PPD of rod-driven synaptic responses in this horizontal cell is attributable to a PPD of release.

To quantify the PPD of release, we measured the exocytotic capacitance change evoked by pairs of stimuli in both rods and cones. Figure 7 illustrates capacitance responses from a rod (Fig. $7 A$ ) and cone (Fig. 7B). Similar to the PSC, the capacitance increase evoked by the second pulse after an interstimulus interval of $100 \mathrm{~ms}$ was much smaller than the original response, but there was nearly complete recovery when the interstimulus interval was lengthened to $1 \mathrm{~s}$. The amplitude of the capacitance increase evoked by the second pulse was divided by the amplitude of the initial response and plotted against the interval between measurements. The time course for recovery was fit with a single exponential function. The capacitance response recovered at nearly identical rates in rods and cones (rods, $\tau=248 \mathrm{~ms}$; cones, $\tau=265 \mathrm{~ms}$; rod/cone difference, $p=0.60 ; F$ test). We also obtained a similar rate of recovery from PPD in cones ( $\tau=256 \mathrm{~ms}$; $n=6$ ) by measuring capacitance changes using a dual sine wave protocol (jClamp; Scisoft, New Haven, CT) instead of the Optopatch phase lock amplifier. PPD produced by interstimulus intervals of 3-30 s remained 3-5\% below baseline (Fig. 7), suggesting that an additional small component to the rate of recovery might be revealed by additional study with long interstimulus intervals.

By comparing Figures 3 and 7, one can see that PPD of exocytosis recovers with a time course that is similar but not identical to the PPD of PSCs. Some disparity between the two measures is expected. Whereas capacitance changes measure the number of vesicles released, PSC amplitude is not a direct measure of glutamate release but is determined by the kinetics of glutamate in the cleft and concentration/response relationships at the receptor. To assess the contribution of presynaptic depression of release to the depression of postsynaptic responses, we compared the recovery from PPD of capacitance and EPSC measurements by linear regression analysis. To do so, we plotted the amount of PPD mea- 
sured with capacitance changes against the amount of PPD measured with PSCs for each interstimulus interval from 0.1 to $3 \mathrm{~s}$ (supplementary Fig., available at www. jneurosci.org as supplemental material). By linear regression on these paired measurements, we obtained correlation coefficients $\left(r^{2}\right)$ ranging from 0.8 to $0.9: r^{2}=$ 0.81 for the correlation between PPD of capacitance changes in rods and PPD of EPSCs in rod-horizontal cell pairs, 0.91 for rod-ON bipolar cell pairs, 0.77 for rod-OFF bipolar cell pairs; $r^{2}=0.85$ for the correlation between PPD of capacitance changes in cones and PPD of EPSCs in cone-horizontal cell pairs, 0.82 for cone-ON bipolar cell pairs, and 0.90 for cone-OFF bipolar cell pairs. Thus, $80-90 \%$ of variance in the PPD of synaptic responses can be accounted for by variance in the PPD of exocytotic capacitance responses.

It has been shown that chloride influx can inhibit endocytosis in bipolar cell terminals (Hull and von Gersdorff, 2004). However, the rate of recovery from PPD of the EPSC was unchanged in both rod$(n=8)$ and cone-driven $(n=2)$ horizon-

tal cells by the addition of niflumic acid $(0.2 \mathrm{~mm}$; data not shown), indicating that blocking calcium-activated chloride channels with niflumic acid during capacitance recordings did not contribute to differences between these two measures in rates of recovery.

We tested PPD with depolarizing steps to $-30 \mathrm{mV}$, close to the dark resting potential for photoreceptors. (Steps to $-40 \mathrm{mV}$ did not produce consistently measurable capacitance responses.) As with steps to $-10 \mathrm{mV}$, exocytosis was depressed by nearly $50 \%$ when steps were separated by an interpulse interval of $100 \mathrm{~ms}$ (rods, $44.0 \pm 5.8 \%, n=7$; cones, $53.4 \pm 9.2 \%, n=9$ ). The rates of recovery with steps to $-30 \mathrm{mV}$ were not significantly different between rods and cones (rods, $\tau=220 \mathrm{~ms}, n=7-10$ for each data point; cones, $\tau=263 \mathrm{~ms}, n=7-9$ for each data point; rod/cone difference, $p=0.55, F$ test). Recovery rates were also not significantly faster than those observed with steps to $-10 \mathrm{mV}$; this may be explained by the fact that $I_{\mathrm{Ca}}$ measured at $-30 \mathrm{mV}$ is still relatively large. Using a ramp voltage protocol $(0.5 \mathrm{mV} / \mathrm{ms})$, the amplitudes of $I_{\mathrm{Ca}}$ measured at $-30 \mathrm{mV}$ in rods and cones were $62 \%(n=19)$ and $47 \%(n=15)$ (Fig. 8), respectively, of $I_{\mathrm{Ca}}$ measured at $-10 \mathrm{mV}$.

Photoreceptor $I_{\mathrm{Ca}}$ exhibits a relatively slow $\mathrm{Ca}^{2+}$-dependent inactivation (Corey et al., 1984; Rabl and Thoreson, 2002), and cone $I_{\mathrm{Ca}}$ can be inhibited by presynaptic type III mGluRs (Hosoi et al., 2005). To assess the contribution of $I_{\mathrm{Ca}}$ inhibition to PPD, we measured $I_{\mathrm{Ca}}$ using the paired-pulse protocol. In both rods and cones, an interpulse interval of $100 \mathrm{~ms}$ depressed the capacitance response much more than it depressed $I_{\mathrm{Ca}}$ (Fig. 8). If there is cooperativity between calcium and release, a small amount of depression in $I_{\mathrm{Ca}}$ can produce a large depression in postsynaptic responses, as found at the calyx of Held ( $\mathrm{Xu}$ and $\mathrm{Wu}, 2005)$. However, unlike the calyx of Held, rod photoreceptors exhibit a linear relationship between $I_{\mathrm{Ca}}$ and exocytotic capacitance changes (Thoreson et al., 2004). Similar to rods, the amplitude of $I_{\mathrm{Ca}}$ recorded from cone photoreceptors using a ramp voltage pro- tocol $(0.5 \mathrm{mV} / \mathrm{ms})$ showed a nearly linear relationship to the charge transfer accompanying horizontal cell EPSCs (reflecting the amount of glutamate released) evoked by depolarizing steps $(100 \mathrm{~ms})$ to different holding potentials (Fig. 8D). $I_{\mathrm{Ca}}$ evoked at $-20 \mathrm{mV}$ is $11 \%$ smaller than that evoked at $-10 \mathrm{mV}$, similar to the $10 \%$ reduction in $I_{\mathrm{Ca}}$ produced by $\mathrm{PPD}$, and this $11 \%$ reduction in $I_{\mathrm{Ca}}$ produces only a $12 \%$ reduction in EPSC charge transfer. Also consistent with a nearly linear relationship between $I_{\mathrm{Ca}}$ and release, the slope of the linear regression relating $I_{\mathrm{Ca}}$ and EPSC charge transfer plotted on $\log / \log$ coordinates was 0.86 $\left(r^{2}=0.98\right)$. Restricting this regression analysis to subsaturating responses evoked by membrane potentials from -50 to $-30 \mathrm{mV}$ yielded a log/log slope of $0.85\left(r^{2}=0.96\right)$, indicating that linearity between $I_{\mathrm{Ca}}$ and EPSC charge transfer is not an artifact of response saturation that might be produced by strong depolarizing stimuli. These findings suggest that, under the conditions of our experiments, PPD of exocytosis in photoreceptors is primarily caused by vesicle depletion, not inhibition of $I_{\mathrm{Ca}}$, consistent with results at other ribbon and conventional synapses (Charlton et al., 1982; von Gersdorff and Matthews, 1997; Schneggenburger et al., 2002).

\section{Discussion}

The present study shows that rods and cones exhibit marked synaptic depression when stimulated with a pair of identical depolarizing pulses. Postsynaptic responses were depressed by $\geq 50 \%$ using an interstimulus interval of $100 \mathrm{~ms}$ and test pulses of $100 \mathrm{~ms}$, long enough to deplete the readily releasable pool of vesicles in rods and cones (Thoreson et al., 2004; Rabl et al., 2005). Both presynaptic and postsynaptic mechanisms can contribute to short-term depression of postsynaptic responses (for review, see Zucker and Regehr, 2002). Postsynaptically, saturation of the intracellular signaling mechanism engaged by the glutamate receptor mGluR6 in ON bipolar cells could potentially contribute to PPD in this cell type (Sampath and Rieke, 2004). 


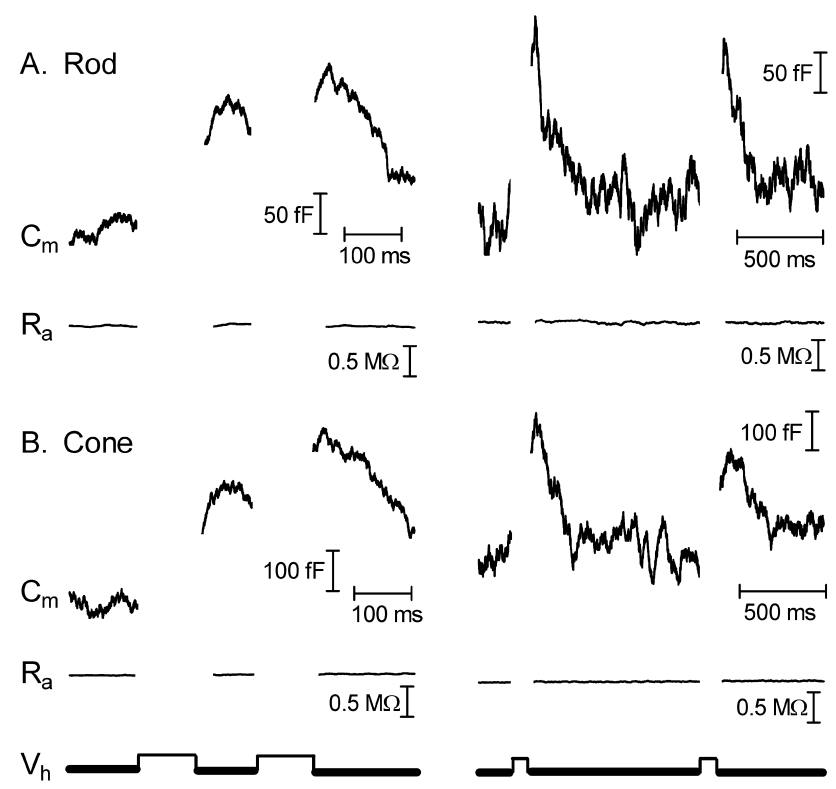

C

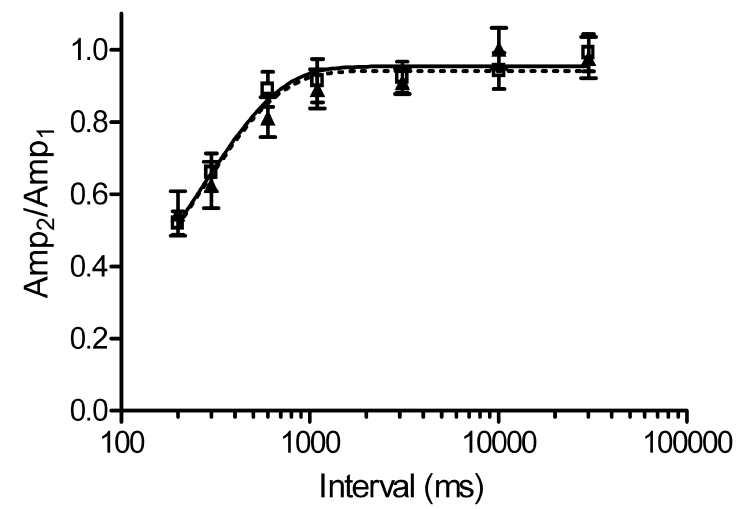

Figure 7. PPD of exocytosis recovered with the same time course in rods and cones. Pairs of capacitance responses were evoked in a rod $(\boldsymbol{A})$ or cone $(\boldsymbol{B})$ by depolarizing pulses $(100 \mathrm{~ms},-70$ to $-10 \mathrm{mV}$ ) applied at $100 \mathrm{~ms}$ (left) or $1000 \mathrm{~ms}$ (right) interpulse intervals. There was no appreciable change in access resistance $\left(R_{\mathrm{a}}\right)$ after the step. Capacitance measurements were suspended during the test step and resumed $3 \mathrm{~ms}$ afterward. $V_{h}$, Holding potential. $C$, Time course for recovery of exocytotic capacitance responses from PPD. The capacitance increase evoked by the second pulse $\left(A \mathrm{Ap}_{2}\right)$ was divided by the amplitude of the first response $\left(\mathrm{Amp}_{1}\right)$ and plotted against the time from the end of the first pulse to the end of the second pulse (i.e., the interval between capacitance measurements). Data were fit with a single exponential function (rods, $\tau=248 \mathrm{~ms}$, open square and solid line; cones, $\tau=265 \mathrm{~ms}$, filled triangles and dashed line; $F$ test comparison of $\tau$ values, $p=0.60)$. Sample sizes: rods: $100 \mathrm{~ms}, n=39 ; 200$ $\mathrm{ms}, n=33 ; 500 \mathrm{~ms}, n=31 ; 1 \mathrm{~s}, n=31 ; 3 \mathrm{~s}, n=23 ; 10 \mathrm{~s}, n=25 ; 30 \mathrm{~s}, n=25 ;$ cones: $100 \mathrm{~ms}$, $n=12 ; 200 \mathrm{~ms}, n=12 ; 500 \mathrm{~ms}, n=13 ; 1 \mathrm{~s}, n=12 ; 3 \mathrm{~s}, n=11 ; 10 \mathrm{~s}, n=17 ; 30 \mathrm{~s}, n=9$. Error bars represent SEM.

Another postsynaptic mechanism that might contribute to PPD is receptor desensitization (Zucker and Regehr, 2002). By using a caged glutamate compound, we showed that horizontal cell AMPA receptors remained only slightly desensitized $500 \mathrm{~ms}$ after an initial pulse of glutamate consistent with relatively rapid recovery of horizontal cell AMPA receptors from desensitization (Eliasof and Jahr, 1997). Reducing desensitization by application of cyclothiazide or use of positive holding potentials had little effect on the recovery from PPD. Thus, in horizontal cells, which showed the strongest PPD of the three postsynaptic cell types, receptor desensitization plays only a minor role in PPD of postsynaptic responses $(\sim 10 \%$ or less with an interstimulus interval of $0.5 \mathrm{~s}$ ). We did not examine a sufficient number of OFF
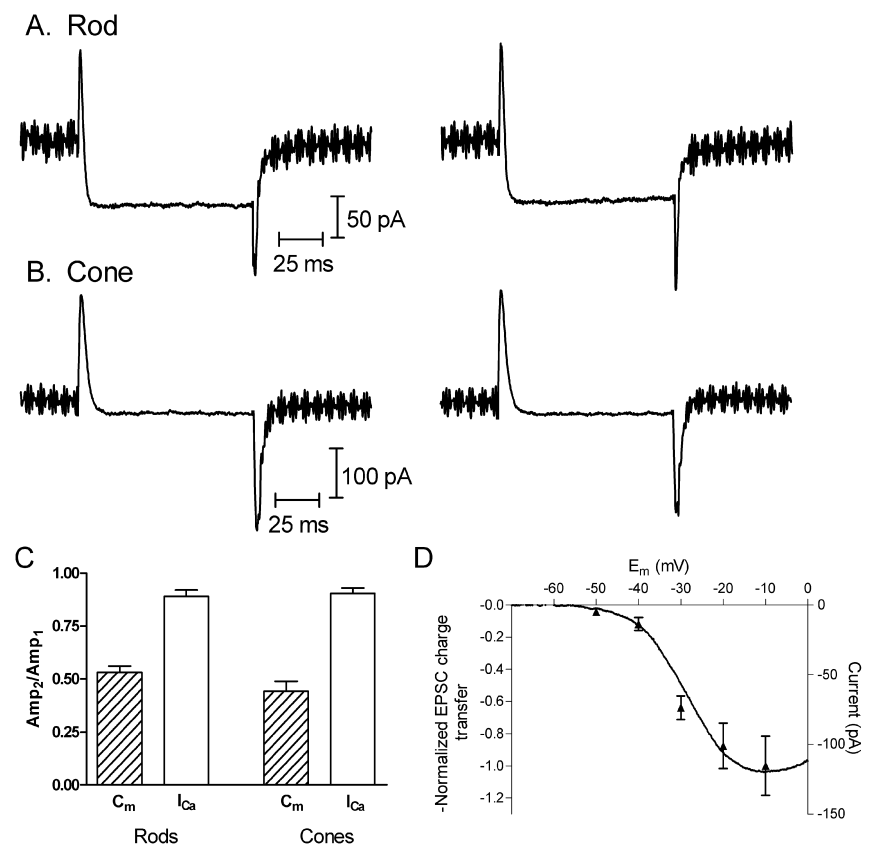

Figure 8. PPD of exocytosis greatly exceeds PPD of $I_{\mathrm{Ca}}$. A, Leak-subtracted $I_{\mathrm{Ca}}$ in a rod evoked by a depolarizing test step $(-70$ to $-10 \mathrm{mV}$ ) before (left) and after (right) an interstimulus interval of $100 \mathrm{~ms}$. The baseline noise before and after the test step is caused by the sinusoidal holding potential used for capacitance measurement. $B, I_{C a}$ in a cone before and after $100 \mathrm{~ms}$ interstimulus interval. $C$, Bar graph comparing the PPD of $I_{C_{a}}$ and the exocytotic capacitance response produced by an interstimulus interval of $100 \mathrm{~ms}$. The amplitude of the second response $\left(A m p_{2}\right)$ was divided by the amplitude of the first response $\left(A m p_{1}\right)$. Capacitance and $I_{\mathrm{Ca}}$ were each measured in nine rods and eight cones. Control $I_{\mathrm{Ca}}$ averaged $70.2 \pm 10.1 \mathrm{pA}$ in rods and $56.2 \pm 7.1 \mathrm{pA}$ in cones. $\boldsymbol{D}$, Overlay of cone $I_{\mathrm{C}_{\mathrm{a}}}$ and the charge transfer of horizontal cell EPSCS evoked by depolarizing steps ( $100 \mathrm{~ms}$ ) applied to simultaneously recorded cones $(n=15)$. Cone $I_{\mathrm{Ca}}$ was measured using a ramp voltage protocol ( -90 to $+60 \mathrm{mV}, 0.5 \mathrm{mV} / \mathrm{ms}$ ) and averaged, after normalizing, from 15 cone photoreceptors. The match between Ca $_{\mathrm{C}}$ and EPSC charge transfer is consistent with a linear relationship between calcium influx and release. Error bars represent SEM.

bipolar cells to determine whether there are extensive cell to cell differences in the properties of desensitization among salamander OFF bipolar cells, as found by DeVries (2000) in ground squirrel retina.

We found greater PPD in the presence of the low-affinity glutamate receptor antagonist kynurenic acid. Greater PPD in the presence of a low-affinity, competitive glutamate antagonist can be interpreted in two ways: (1) greater PPD can be produced by a more efficacious block of the second EPSC by kynurenic acid. If so, this would suggest that a lower concentration of glutamate is attained in the synaptic cleft during the second EPSC (Hashimoto and Kano, 1998; Wadiche and Jahr, 2001; Shen et al., 2002). Such a situation can occur if there is multivesicular release (Wadiche and Jahr, 2001), as found at the bipolar cell ribbon synapse (Singer et al., 2004). (2) If postsynaptic receptors are saturated by glutamate levels in the cleft during the initial EPSC, then relieving saturation with kynurenic acid will lead to greater PPD and reveal a more accurate portrayal of the underlying changes in release (Foster et al., 2002; Harrison and Jahr, 2003). Two pieces of evidence suggest that glutamate receptors at photoreceptor synapses are not saturated during EPSCs evoked by steps to $-10 \mathrm{mV}$ : (1) there is a linear correlation between exocytotic capacitance changes and EPSC charge transfer in both rods and cones for depolarizing test steps ranging in duration from a few millisecond to seconds (Thoreson et al., 2004; Rabl et al., 2005). This result suggests that postsynaptic receptors are not saturated but 
are capable of sensing the release of additional glutamate evoked by longer test pulses. (2) The waveforms of EPSCs evoked by presynaptic stimulation of photoreceptors can be reproduced by convolving individual miniature EPSCs (mEPSCs) with the presynaptic release function (Cadetti et al., 2005). This suggests that every mEPSC contributes equally to the overall EPSC. Postsynaptic receptors at the bipolar cell ribbon synapse also do not appear be saturated by synaptically released glutamate (Singer and Diamond, 2003). From these results suggesting that glutamate receptors are not saturated, it seems more likely that the greater PPD observed in kynurenic acid is because of a stronger block of the second EPSC arising from a lower concentration of glutamate in the synaptic cleft (Hashimoto and Kano, 1998; Wadiche and Jahr, 2001). We also directly examined depression of presynaptic release using capacitance measurements. Correlations between the recovery from PPD of presynaptic capacitance changes and the recovery of postsynaptic responses suggest $80-$ $90 \%$ of the PPD of PSCs is caused by a presynaptic depression of release.

Presynaptic mechanisms that contribute to PPD of postsynaptic responses include depression of $I_{\mathrm{Ca}}$ and depletion of the pool of releasable vesicles. In rod photoreceptors, depolarizationevoked inhibition of $I_{\mathrm{Ca}}$ can be produced by $\mathrm{Ca}^{2+}$-dependent inactivation (Corey et al., 1984; Rabl and Thoreson, 2002) as well as the depletion of synaptic cleft $\mathrm{Ca}^{2+}$ levels (Rabl and Thoreson, 2002). In cones, there is also negative feedback inhibition of $I_{\mathrm{Ca}}$ by vesicular protons and metabotropic glutamate receptors (DeVries, 2001; Hosoi et al., 2005). The block of cone $I_{\mathrm{Ca}}$ by vesicular protons recovered from PPD with the same time course as postsynaptic responses in mammalian OFF bipolar cells, suggesting that this mechanism is rate limiting in short-term depression at this synapse (DeVries, 2001). Under our recording conditions with HEPES as a $\mathrm{pH}$ buffer, the short-term depression of $I_{\mathrm{Ca}}$ produced with a paired-pulse protocol was minimal (Fig. 8). The nearly linear relationship between calcium influx and release at rod and cone synapses (Fig. 8) (Thoreson et al., 2004) implies that the reduction of $\sim 10 \%$ in $I_{\mathrm{Ca}}$ accounts for one-fifth of the $45-$ $55 \%$ depression of release observed with an interstimulus interval of $100 \mathrm{~ms}$. The remaining depression of release is likely caused by a depletion of presynaptic vesicles, as found at other ribbon and conventional synapses (Charlton et al., 1982; von Gersdorff and Matthews, 1997; Schneggenburger et al., 2002; von Gersdorff and Borst, 2002). Depolarizing steps to $-40 \mathrm{mV}$ applied to rods produce less $\mathrm{Ca}^{2+}$ influx, and consequently less release, than steps to $-10 \mathrm{mV}$. Consistent with the suggestion that synaptic depletion is a major contributor to PPD at photoreceptor synapses, horizontal cells recovered from PPD more rapidly when fewer vesicles were depleted by using steps to $-40 \mathrm{mV}$ than by using steps to $-10 \mathrm{mV}$ (Fig. 5).

Because of gap junction coupling between rods (Attwell et al., 1984), depolarizing current injected into one rod can stimulate exocytosis from neighboring rods contributing to later portions of the rod-driven PSC (Cadetti et al., 2005). Cones exhibit little coupling (Attwell et al., 1984), and thus coupling would be predicted to have more significant effects in rod-driven cells than cone-driven cells. Unlike paired recordings, exocytotic capacitance changes are not influenced by release from coupled rods. If coupling had a significant impact on the rate at which PSCs recover from PPD, then there should be significant rod/cone differences measured with paired recordings but not with capacitance recordings. However, rods and cones recovered at similar rates whether PPD was measured by capacitance or paired re- cordings, suggesting that coupling had little impact on the rate of PSC recovery from PPD.

Rates of recovery from PPD vary widely from synapse to synapse. At some synapses, replenishment of presynaptic vesicles is so rapid that PPD is not detectable, whereas full recovery at other synapses can take minutes (Zucker and Regehr, 2002). There are multiple kinetic components to recovery from PPD at ribbon synapses of cochlear hair cells and retinal bipolar cells, and complete recovery can take seconds (von Gersdorff and Matthews, 1997; Burrone and Lagnado 2000; Moser and Beutner, 2000; Eisen et al., 2004). The recovery of responses achieved by photoreceptors in the present study exhibited a relatively rapid time course that could be approximated by a single exponential $(\tau \sim$ $250 \mathrm{~ms}$ ). Some of the differences in recovery rates between photoreceptors and other ribbon synapses appear to be because of differences in experimental conditions. One factor is calcium buffering: the presence of $5 \mathrm{~mm}$ EGTA in the patch-pipette solution, as used in the present study, has been shown to inhibit a slow component of recovery from PPD of exocytotic capacitance changes in bipolar cell terminals (Gomis et al., 1999). A second important factor is the test pulse amplitude and duration because this determines the amount of vesicular release and thus presynaptic depletion. Using a test pulse (100 ms, -70 to $-20 \mathrm{mV}$ ) similar to that in the present study, interstimulus intervals of 100 and $200 \mathrm{~ms}$ produced PPD of 46 and 29\%, respectively, in the capacitance response of hair cells (Edmonds et al., 2004), quite similar to the depression of 45 and 33-35\% observed in the rods and cones with the same interstimulus intervals.

The kinetics of vesicular release and replenishment helps to shape the synaptic transfer function and contribute to a decrease in synaptic transmission from photoreceptors to second-order cells at high temporal frequencies. Recovery from PPD of the exocytotic capacitance response, which primarily reflects vesicle replenishment, exhibits a time constant of $\sim 250 \mathrm{~ms}$ and may therefore contribute to the decrease in synaptic output from amphibian photoreceptors at frequencies above $4 \mathrm{~Hz}$ (ArmstrongGold and Rieke, 2003). Although vesicle replenishment rates may help to shape the frequency response of synaptic transmission, the absence of rod/cone differences in vesicle replenishment rates indicate that other factors (e.g., differences in rates of exocytosis) are responsible for differences in the frequency response between rod and cone synapses (Pasino and Marchiafava, 1976; Schnapf and Copenhagen, 1982).

\section{References}

Armstrong-Gold CE, Rieke F (2003) Band-pass filtering at the rod to second-order cell synapse in salamander (Ambystoma tigrinum) retina. J Neurosci 23:3796-3806.

Attwell D, Wilson M, Wu SM (1984) A quantitative analysis of interactions between photoreceptors in the salamander (Ambystoma) retina. J Physiol (Lond) 352:703-737.

Bellingham MC, Walmsley B (1999) A novel presynaptic inhibitory mechanism underlies paired pulse depression at a fast central synapse. Neuron 23:159-170.

Burrone J, Lagnado L (2000) Synaptic depression and the kinetics of exocytosis in retinal bipolar cells. J Neurosci 20:568-578.

Cadetti L, Thoreson WB (2006) Feedback effects of horizontal cell membrane potential on cone calcium currents studied with simultaneous recordings. J Neurophysiol, in press.

Cadetti L, Tranchina D, Thoreson WB (2005) A comparison of release kinetics and glutamate receptor properties in shaping rod-cone differences in EPSC kinetics. J Physiol (Lond) 569:773-788.

Charlton MP, Smith SJ, Zucker RS (1982) Role of presynaptic calcium ions and channels in synaptic facilitation and depression at the squid giant synapse. J Physiol (Lond) 323:173-193. 
Clements JD (1996) Transmitter timecourse in the synaptic cleft: its role in central synaptic function. Trends Neurosci 19:163-171.

Corey DP, Dubinsky JM, Schwartz EA (1984) The calcium current in inner segments of rods from the salamander (Ambystoma tigrinum) retina. J Physiol (Lond) 354:557-575.

DeVries SH (2000) Bipolar cells use kainate and AMPA receptors to filter visual information into separate channels. Neuron 28:847-856.

DeVries SH (2001) Exocytosed protons feedback to suppress the $\mathrm{Ca}^{2+}$ current in mammalian cone photoreceptors. Neuron 32:1107-1117.

Diamond JS, Jahr CE (1995) Asynchronous release of synaptic vesicles determines the time course of the AMPA receptor-mediated EPSC. Neuron 15:1097-1107.

Edmonds BW, Gregory FD, Schweizer FE (2004) Evidence that fast exocytosis can be predominantly mediated by vesicles not docked at active zones in frog saccular hair cells. J Physiol (Lond) 560:439-450.

Eisen MD, Spassova M, Parsons TD (2004) Large releasable pool of synaptic vesicles in chick cochlear hair cells. J Neurophysiol 91:2422-2428.

Eliasof S, Jahr CE (1997) Rapid AMPA receptor desensitization in catfish cone horizontal cells. Vis Neurosci 14:13-18.

Foster KA, Kreitzer AC, Regehr WG (2002) Interaction of postsynaptic receptor saturation with presynaptic mechanisms produces a reliable synapse. Neuron 36:1115-1126.

Gomis A, Burrone J, Lagnado L (1999) Two actions of calcium regulate the supply of releasable vesicles at the ribbon synapse of retinal bipolar cells. J Neurosci 19:6309-6317.

Harrison J, Jahr CE (2003) Receptor occupancy limits synaptic depression at climbing fiber synapses. J Neurosci 23:377-383.

Hashimoto K, Kano M (1998) Presynaptic origin of paired-pulse depression at climbing fibre-Purkinje cell synapses in the rat cerebellum. J Physiol (Lond) 506:391-405.

Hirasawa H, Shiells R, Yamada M (2002) A metabotropic glutamate receptor regulates transmitter release from cone presynaptic terminals in carp retinal slices. J Gen Physiol 119:55-68.

Hosoi N, Arai I, Tachibana M (2005) Group III metabotropic glutamate receptors and exocytosed protons inhibit L-type calcium currents in cones but not in rods. J Neurosci 25:4062-4072.

Hull C, von Gersdorff H (2004) Fast endocytosis is inhibited by GABAmediated chloride influx at a presynaptic terminal. Neuron 44:469-482.

Johnson SL, Thomas MV, Kros CJ (2002) Membrane capacitance measurement using patch clamp with integrated self-balancing lock-in amplifier. Pflügers Arch 443:653-663.

Kusano K, Landau EM (1975) Depression and recovery of transmission at the squid giant synapse. J Physiol (Lond) 245:13-22.

Maple BR, Gao F, Wu SM (1999) Glutamate receptors differ in rod- and cone-dominated off-center bipolar cells. NeuroReport 10:3605-3610.

Moser T, Beutner D (2000) Kinetics of exocytosis and endocytosis at the cochlear inner hair cell afferent synapse of the mouse. Proc Natl Acad Sci USA 97:883-888.

Nawy S, Jahr CE (1990) Suppression by glutamate of cGMP-activated conductance in retinal bipolar cells. Nature 346:269-271.

Otis TS, Wu Y-C, Trussell LO (1996a) Delayed clearance of transmitter and the role of glutamate transporters at synapses with multiple release sites. J Neurosci 16:1634-1644.

Otis TS, Zhang S, Trussell LO (1996b) Direct measurement of AMPA receptor desensitization induced by glutamatergic synaptic transmission. J Neurosci 16:7496-7504.
Partin KM, Patneau DK, Winters CA, Mayer ML, Buonanno A (1993) Selective modulation of desensitization at AMPA versus kainate receptors by cyclothiazide and concanavalin A. Neuron 11:1069-1082.

Pasino E, Marchiafava PL (1976) Transfer properties of rod and cone cells in the retina of the tiger salamander. Vision Res 16:381-386.

Rabl K, Thoreson WB (2002) Calcium-dependent inactivation and depletion of synaptic cleft calcium ions combine to regulate rod calcium currents under physiological conditions. Eur J Neurosci 16:2070-2077.

Rabl K, Cadetti L, Thoreson WB (2005) Kinetics of exocytosis is faster in cones than rods. J Neurosci 25:4633-4640.

Raman IM, Trussell LO (1995) Concentration-jump analysis of voltagedependent conductances activated by glutamate and kainate in neurons of the avian cochlear nucleus. Biophys J 69:1868-1879.

Rozov A, Jerecic J, Sakmann B, Burnashev N (2001) AMPA receptor channels with long-lasting desensitization in bipolar interneurons contribute to synaptic depression in a novel feedback circuit in layer $2 / 3$ of rat neocortex. J Neurosci 21:8062-8071.

Sampath AP, Rieke F (2004) Selective transmission of single photon responses by saturation at the rod-to-rod bipolar synapse. Neuron 41:431-443.

Schnapf JL, Copenhagen DR (1982) Differences in the kinetics of rod and cone synaptic transmission. Nature 296:862-864.

Schneggenburger R, Sakaba T, Neher E (2002) Vesicle pools and short-term synaptic depression: lessons from a large synapse. Trends Neurosci 25:206-212.

Shen Y, Hansel C, Linden DJ (2002) Glutamate release during LTD at cerebellar climbing fiber-Purkinje cell synapses. Nat Neurosci 5:725-726.

Singer JH, Diamond JS (2003) Sustained $\mathrm{Ca}^{2+}$ entry elicits transient postsynaptic currents at a retinal ribbon synapse. J Neurosci 23:10923-10933.

Singer JH, Lassova L, Vardi N, Diamond JS (2004) Coordinated multivesicular release at a mammalian ribbon synapse. Nat Neurosci 7:826-833.

Thoreson WB, Nitzan R, Miller RF (1997) Reducing extracellular chloride suppresses dihydropyridine-sensitive calcium currents and synaptic transmission in amphibian photoreceptors. J Neurophysiol 77:2175-2190.

Thoreson WB, Rabl K, Townes-Anderson E, Heidelberger R (2004) A highly $\mathrm{Ca}^{2+}$-sensitive pool of vesicles contributes to linearity at the rod photoreceptor ribbon synapse. Neuron 42:595-605.

von Gersdorff H, Borst JG (2002) Short-term plasticity at the calyx of held. Nat Rev Neurosci 3:53-64.

von Gersdorff H, Matthews G (1997) Depletion and replenishment of vesicle pools at a ribbon-type synaptic terminal. J Neurosci 17:1919-1927.

Wadiche JI, Jahr CE (2001) Multivesicular release at climbing fiberPurkinje cell synapses. Neuron 32:301-313.

Wu LG, Borst JG (1999) The reduced release probability of releasable vesicles during recovery from short-term synaptic depression. Neuron 23:821-832.

$\mathrm{Xu} \mathrm{J}, \mathrm{Wu}$ LG (2005) The decrease in the presynaptic calcium current is a major cause of short-term depression at a calyx-type synapse. Neuron 46:633-645.

Yang JH, Maple B, Gao F, Maguire G, Wu SM (1998) Postsynaptic responses of horizontal cells in the tiger salamander retina are mediated by AMPA-preferring receptors. Brain Res 797:125-134.

Zucker RS, Regehr WG (2002) Short-term synaptic plasticity. Annu Rev Physiol 64:355-405. 